\title{
A correlation-based MIMO Performance Model for the System Level Analysis on MAC layer
}

\author{
Rainer Schoenen ${ }^{1}$ and Carlos Teijeiro, \\ Communication Networks (ComNets), Faculty 6, RWTH Aachen University, Germany \\ rs@comnets.rwth-aachen.de
}

\begin{abstract}
IMT-Advanced systems are currently in their evaluation phase. Due to the complexity of all the integrated algorithms and the multidimensional variability of the wireless channels a system evaluation is not easy and only very few large enterprises have the manpower to construct software to simulate all relevant effects. Traditionally the effects of channel and physical layer algorithms have been studied with bit-level algorithms on a single link. So are most of the results for MIMO processing. System level studies - on the other hand - take cellular layouts, multiple cells and users, real traffic and OSI layers 2 and above into account. They are able to deliver spectral efficiency results. There is a huge gap between these approaches. In order for system level models to give results within reasonable time there must be an abstraction of lower layer details. In this paper a simplified MIMO performance model is presented which can calculate the resource capacity in $O(M)$ and has one parameter which allows to adjust the MIMO capability of a link. The model is then applied to the numeric evaluation of LTE in IMT-Advanced scenarios.
\end{abstract}

Index Terms-IMT-Advanced, LTE, Relays, MIMO

\section{INTRODUCTION}

$\mathbf{M}$ IMO (multiple input multiple output) is a technology positioned in every cellular standard of the future because the high goals of IMT-Advanced [1] can only be achieved by using the extra spatial dimension. From experiences with IEEE802.11 home networks we all intuitively know that this is going to work well only in regions where the signal-to-(interference+noise) level $S I N R$ is high enough. In reuse-1 scenarios the interferer locations (base stations, BS) are in highest packing density, so at the cell or sector edges there will be an expected $S I N R$ of zero which requires different techniques to mitigate.

Using Fixed Relay Stations (FRS), also called Relay Nodes $(\mathrm{RN})$, is one solution [2]-[4]. Another is a variety of interference coordination schemes, e.g. dynamic or fractional frequency reuse [5]. Some believe that cooperative transmission brings benefits [6] but due to the enormous overhead in interBS signaling and MAC-layer effort there can be doubts about it in practice [7].

For the evaluation of these future technologies there is a huge effort required because of the many effects and algorithms which have to be considered. Only few corporate companies have the manpower for programming extensive simulation studies, while it becomes harder and harder for universities to contribute substantially [7].

When is comes to system level analysis, this typically requires studying scenarios with 57 cell sectors, 570 user

\footnotetext{
${ }^{1}$ Dr. Schoenen is spending 2010 at Carleton University, Canada, collaborating with Dr. Yanikomeroglu
}

terminals (UT) [8] and a lot of parameters that influence the performance. Simulation studies usually require a huge number of drops (experiment with random placement of UTs) to converge to the average results required. That is why the use of detailed channel models and bit-accurate problem formulations are prohibitive in large studies.

In this paper we propose a model for the SU-MIMO performance depending on SINR and an antenna correlation parameter $c$ only. This basic stochastic model calculates the metrics in $O(M)$ during (simulation|analysis) runtime, where $M$ is the number of transmit antennas. Other more complex performance models require operations like FFT, matrix inversion etc and are not cheaper than $O\left(M^{3}\right)$. The model is independent of the underlying random process, so there can be a correlation in time if required to capture these effects [9].

Based on the MIMO-SINR, MI and PER calculations the system capacity can be calculated. For basic SISO scenarios this has been investigated in [10] while some IMTAdvanced scenarios [8] were focused in [11].

The paper is organized as follows. The first section gives an overview of the performance models used. The system level (large scale) MIMO model is explained next. Then the properties and model parameter tables are presented. The model is then applied to an IMT-Advanced scenario case and performance results for SISO and MIMO are compared. A concluding summary reformulates the contributions in this paper.

\section{SYSTEM LEVEL PERFORMANCE MODELS}

In order to determine higher layer system level results with reasonable time and effort, there must be an abstraction from the physics to mathematical models suitable of capturing all relevant effects. One important aspect in recent years is the (simulation|analysis) efficiency as this decides whether meaningful results can be obtained using all implemented algorithms or not. Currently simulations require around 100 drops with $T=10 \mathrm{~s}$ simulated time which are roughly 1000000 frame samples. Within each frame 57 BSs and 570 UTs are considered, and a total of $C=100$ OFDMA subchannels per frame, i.e. 57000 resource options. Each scheduling decision may take $O(C)$ to $O\left(C^{2}\right)$. Only the MAC layer is responsible for resource and packet scheduling [12].

For each resource usage opportunity the $\operatorname{SINR}\left(U T_{u}\right)$ has to be determined (Downlink=DL: one server and 56 interferers). For MIMO there is one effective $\operatorname{SIN}_{l}\left(U T_{u}\right)$ per stream $l$ and user $u$. Based upon this, an Adaptive 


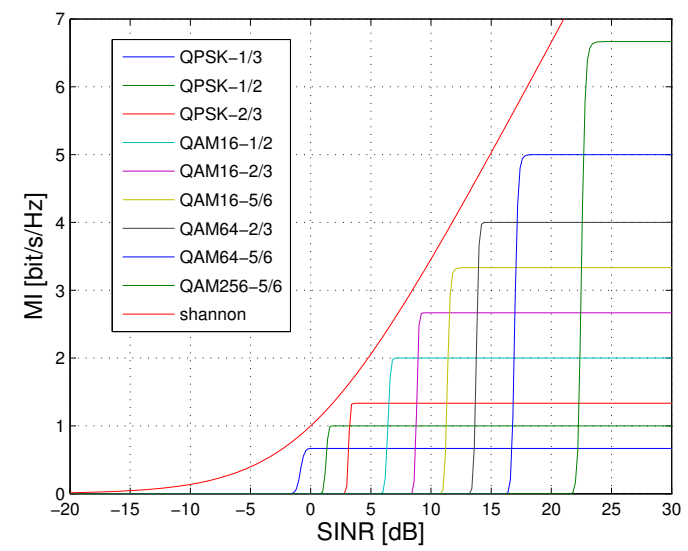

Fig. 1. Link level performance (net $M I$ ) for different modulation\&coding schemes (PhyModes). QAM256 is not used here.

TABLE I

PHYMODES AND SINR INTERVALS

\begin{tabular}{|c|c|c|c|c|c|c|c|c|}
\hline SINR & 0.9 & 2.1 & 3.8 & 7.7 & 9.8 & 12.6 & 15.0 & 18.2 \\
\hline Mod. & \multicolumn{3}{|c|}{ QPSK } & \multicolumn{3}{|c|}{ QAM16 } & \multicolumn{2}{|c|}{ QAM64 } \\
\hline Cod. & $1 / 3$ & $1 / 2$ & $2 / 3$ & $1 / 2$ & $2 / 3$ & $5 / 6$ & $2 / 3$ & $5 / 6$ \\
\hline
\end{tabular}

Modulation and Coding (AMC) unit determines the PhyMode (modulation\&coding scheme) in this resource (different per stream l). See Fig. 1 for the mapping curves. The PhyModes are chosen according to Tab. I. The model is based on the steps $S I N R \rightarrow M I, M I \rightarrow B E R$ and $B E R \rightarrow P E R$ to get the packet error probability [13] [14]. The ARQ performance determines $P E R$, which reduces the effective $M I$ and so the average net bit capacity (bits/s/Hz) is obtained.

$$
M I_{\text {above } A R Q}=M I_{\text {below } A R Q} \cdot(1-P E R)
$$

A pathloss model captures the power reduction as a function of the distance $d(B S-U T)$ (propagation). The IMTAdvanced evaluation standard models are considered useful. The detailed parameters depend on the scenario (Tab. V) and can be found in [8]. One important aspect of these pathloss models is that there is a probability function $p_{L O S}(d)$ to decide the likelihood of line-of-sight (LOS) or non-LOS (NLOS) at a position in $d$. Antenna downtilt is considered standard these days (Tab. V). The rest of the calculation goes as described here and a typical example scenario for $P_{R x}$ and $P_{I f}$ is shown in Fig. 8.

- Transmit Power $P_{T x}$ : see Table V,

- Pathloss: see Table V and [8] [11],

- Interference I: neighbor cell BSs and neighbor sectors interfere ( $100 \%$ load, cluster order 1)

- Noise $N$ : accounted for but not serious ( $I$-limited),

- SINR: $S I N R=S /(N+I)$,

- MI: mutual information $M I=f(S I N R, \bmod )$ [14],

- BER: bit error ratio, depends on $M I$,

- PER: packet error ratio, the result after channel decoding,

- Throughput: determined by bandwidth, PhyMode (modulation and code rate), ARQ overhead,

- Spectral Efficiency: net $M I[b i t / s / H z]$ is throughput per bandwidth averaged over the cell (sector) area [14],

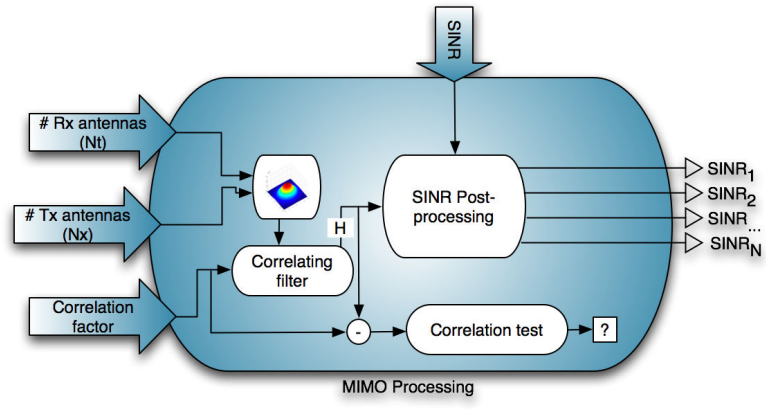

Fig. 2. Obtaining spatial SINR results for MIMO

- Relays: least resources BS/RN association [14],

\section{A SYSTEM-LEVEL MIMO PERFORMANCE MODEL}

For a single antenna system it was sufficient to consider pathloss, antenna gain, fading, shadowing and interference only to obtain the SINR at the receiver. For MIMO there are spatial layers and MIMO receivers output received signals for each of these layers separately. Each spatial layer $l$ has its own $S I N R_{l}$ experience. The $S I N R_{l}$ are proportional to the SINR $R_{S I S O}$ calculated for SISO, because pathloss considerations are the same, but due to physical effects each stream $l$ is different and usually lower than the $S I N R_{S I S O}$ (see Fig. 3). For SU-MIMO each spatial stream has its own PhyMode depending on $S I N R_{l}$ using the same mapping as in section II. Full channel quality information (CQI) must be assumed. By having $M$ spatial streams altogether, the performance of MIMO transmission goes beyond SISO, even though typically all $S I N R_{l}$ are lower than $S I N R_{S I S O}$ :

$$
M I_{\text {sum }}=\sum_{i=1}^{M} M I\left(S I N R_{l}\right)
$$

The postprocessing $S I N R_{l}$ is determined according to [15], [16], where the post-processing $S I N R_{l}$ on stream $l$ is given by Eq. 3. The right term is called $\triangle S I N R$, because in the $\mathrm{dB}$ domain is is just added to the $S I N R_{S I S O}$ :

$$
S I N R_{l}=\frac{E}{M_{t} N_{0}} \frac{1}{\left[\mathbf{H}^{H} \mathbf{H}\right]_{l, l}^{-1}}=\frac{S I N R_{l}}{M_{t}} \frac{1}{\left[\mathbf{H}^{H} \mathbf{H}\right]_{l, l}^{-1}}
$$

where $\mathbf{H}_{l, l}$ stands for the $(l, l)$ entry of the channel matrix H. Usually the spatial streams appear sorted from highest to lowest $S I N R_{l}$. Typical distributions of $S I N R_{l}$ are shown in fig. 3 .

Figure 2 explains the approach used here. There is just one parameter $c$ that characterizes the degree of independence between the spatial streams. $c$ has the meaning of a correlation factor $c$, since a higher antenna correlation leads to less MIMO performance gain. $c$ is used to construct a covariance matrix $R$, where all but the diagonal elements are filled with $c$. An eigenvalue decomposition of $R$ leads to the diagonal matrix $D$ (and the corresponding eigenvectors in the columns of a matrix $V$ ) according to Eq. 4.

$$
\mathbf{R}=\mathbf{V}_{\mathbf{R}} \mathbf{D}_{\mathbf{R}} \mathbf{V}_{\mathbf{R}}{ }^{H}
$$

From there we obtain a correlating filter $\mathbf{W}$ :

$$
\mathbf{W}=\mathbf{V}_{\mathbf{R}} \sqrt{\mathbf{D}_{\mathbf{R}}}
$$




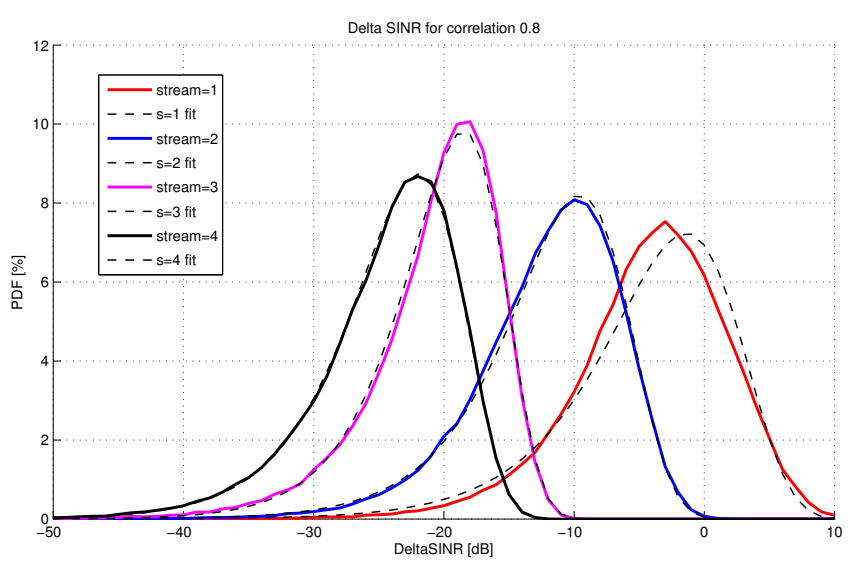

Fig. 3. PDF of post-processing $\Delta S I N R_{l}$ for $c=0.8$ and its PDF fit

Then the filter $\mathbf{W}$ is applied to the totally uncorrelated ("white" Gaussian) channel matrix $\mathbf{H}_{\mathbf{w}}$ to obtain the correlated channel matrix in Eq. 6 needed for Eq. 3. For validation purposes, according to Fig. 4 the correlation of the outcome was also measured and the comparison of input and output showed a very good match.

$$
\mathbf{H}=\mathbf{W H}_{\mathbf{w}}
$$

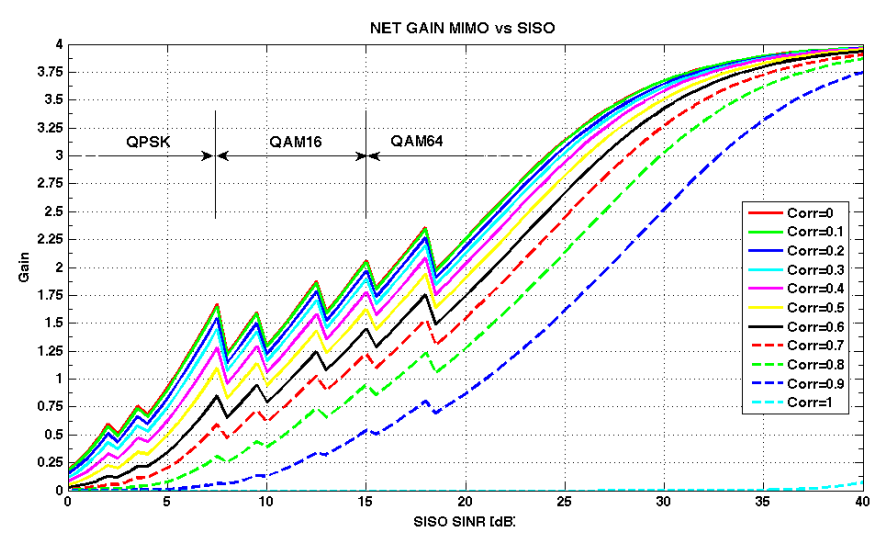

Fig. 4. Net gain between MIMO and SISO $M I$ in $[$ bits $/ s / H z]$ depending on antenna correlation parameter

In Fig. 4 the net MIMO gain has been obtained by dividing $M I_{\text {sum }}$ of Eq. 2 by the $M I_{S I S O}$. It can be seen from Eq. 3 to 6 that this is a computationally intensive calculation. It appears as prohibitive for efficient system-level studies. The solution to more efficiency is the observation that this random experiment always produces results for $\triangle S I N R_{l}$ according to the same random distribution (Fig. 3). The probability density function (PDF) of these $\Delta S I N R_{l}$ is well described with an Extreme Value Distribution. Eq. 7 describes it completely. Figure 3 shows the fitting result.

$$
P(x \mid \mu, \sigma)=\frac{1}{\sigma} \cdot \exp \left(\frac{x-\mu}{\sigma}\right) \cdot \exp \left(-\exp \left(\frac{x-\mu}{\sigma}\right)\right)
$$

So the random values $\Delta S I N R_{l}$ in Fig. 3 can also be obtained by drawing $M$ individual random numbers according to this distribution (Eq. 7), but with different parameters for each $l$. The parameters $\mu(l)$ and $\sigma(l)$ must now be determined as a
TABLE II

LOOKUP TABLE FOR THE PARAMETERS OF EQ. 7 IN MIMO- $4 x 4$

\begin{tabular}{|l||c|c|c|c|c|c|c|c|}
\hline \multicolumn{1}{l|}{$l=$} & \multicolumn{2}{|c|}{ stream 1 } & \multicolumn{2}{c|}{ stream 2 } & \multicolumn{2}{c|}{ stream 3 } & \multicolumn{2}{c|}{ stream 4 } \\
$c=$ & $\mu$ & $\sigma$ & $\mu$ & $\sigma$ & $\mu$ & $\sigma$ & $\mu$ & $\sigma$ \\
\hline \hline 0.0 & -3.0469 & 3.1890 & -5.4853 & 3.4699 & -7.3924 & 3.7639 & -9.5372 & 4.1188 \\
0.1 & -2.9540 & 3.2851 & -5.5353 & 3.4964 & -7.5439 & 3.7738 & -9.7900 & 4.1471 \\
0.2 & -2.7110 & 3.5083 & -5.6849 & 3.5663 & -7.9713 & 3.7792 & -10.4524 & 4.1557 \\
0.3 & -2.4177 & 3.7634 & -5.9065 & 3.6839 & -8.6737 & 3.7695 & -11.4037 & 4.1773 \\
0.4 & -2.1261 & 3.9960 & -6.2190 & 3.8257 & -9.6538 & 3.7629 & -12.6066 & 4.1967 \\
0.5 & -1.8535 & 4.2562 & -6.6321 & 4.0025 & -10.9633 & 3.7498 & -14.1232 & 4.1902 \\
0.6 & -1.6702 & 4.4636 & -7.2397 & 4.1572 & -12.7054 & 3.7389 & -16.0112 & 4.1894 \\
0.7 & -1.4943 & 4.7703 & -8.1390 & 4.3193 & -15.0754 & 3.7448 & -18.4758 & 4.2051 \\
0.8 & -1.4138 & 5.0833 & -9.5362 & 4.4796 & -18.4893 & 3.7412 & -21.9932 & 4.2123 \\
0.9 & -1.4315 & 5.5264 & -12.2424 & 4.5883 & -24.4648 & 3.7302 & -28.0236 & 4.2040 \\
1.0 & -1.6232 & 6.2630 & -41.9751 & 4.7659 & -84.4148 & 3.7347 & -87.9965 & 4.2082 \\
\hline
\end{tabular}

TABLE III

LOOKUP TABLE FOR EQ. 7 IN $3 x 3$ AND $2 x 2$

\begin{tabular}{|c|c|c|c|c|c|c|c|c|c|c|}
\hline$M=$ & \multicolumn{4}{|c|}{$3 \times 3$} & \multicolumn{3}{c|}{} & \multicolumn{4}{|c|}{$2 \times 2$} \\
$l=$ & stream 1 & \multicolumn{2}{c|}{ stream 2} & \multicolumn{2}{c|}{ stream 3} & \multicolumn{2}{c|}{ stream 1 } & \multicolumn{2}{c|}{ stream 2} \\
$c=$ & $\mu$ & $\sigma$ & $\mu$ & $\sigma$ & $\mu$ & $\sigma$ & $\mu$ & $\sigma$ & $\mu$ & $\sigma$ \\
\hline \hline 0.0 & -2.3444 & 3.4088 & -5.1500 & 3.7584 & -7.7150 & 4.1628 & -1.3726 & 3.7249 & -4.9907 & 4.2012 \\
0.1 & -2.2698 & 3.4585 & -5.1763 & 3.7481 & -7.8497 & 4.1449 & -1.4204 & 3.7743 & -5.1030 & 4.2066 \\
0.2 & -2.2046 & 3.6178 & -5.4180 & 3.8063 & -8.3726 & 4.1861 & -1.4492 & 3.8328 & -5.3775 & 4.2061 \\
0.3 & -2.0194 & 3.8178 & -5.7064 & 3.8704 & -9.0794 & 4.2371 & -1.4953 & 3.9625 & -5.8351 & 4.2396 \\
0.4 & -1.8890 & 4.0200 & -6.1525 & 3.9702 & -10.1217 & 4.2391 & -1.6617 & 4.1150 & -6.6089 & 4.2860 \\
0.5 & -1.7509 & 4.2583 & -6.7248 & 4.0946 & -11.4283 & 4.2678 & -1.8609 & 4.3121 & -7.5832 & 4.3876 \\
0.6 & -1.6729 & 4.4818 & -7.5215 & 4.2466 & -13.1971 & 4.2902 & -2.2415 & 4.4943 & -8.9700 & 4.4974 \\
0.7 & -1.6774 & 4.7558 & -8.6170 & 4.4234 & -15.5071 & 4.2776 & -2.8361 & 4.7843 & -10.8876 & 4.6850 \\
0.8 & -1.7745 & 5.1151 & -10.3229 & 4.6685 & -18.9270 & 4.3302 & -3.8795 & 5.0926 & -13.7362 & 4.9605 \\
0.9 & -1.9927 & 5.5894 & -13.3315 & 4.9464 & -24.8574 & 4.3258 & -6.0628 & 5.6204 & -18.9968 & 5.4664 \\
1.0 & -2.6144 & 6.6507 & -43.6132 & 5.5718 & -84.7726 & 4.3335 & -34.6578 & 7.8798 & -77.6517 & 7.8215 \\
\hline
\end{tabular}

function of $M$, the stream index $l$ and the correlation factor $c$. But they don't depend on anything else, so they can be calculated a-priori and later be used as a lookup table. The next section provides the numeric results.

\section{BASIC MIMO STOCHASTIC PROCESS}

The random process of generating $\mathbf{H}_{\mathrm{w}}$ was repeatedly applied and according to Eq. 3 to 6 the $S I N R_{l}$ were obtained for 10000 samples. A distribution fit to Eq. 7 was calculated per $l$ given the histogram of all $S I N R_{l}$. The result can be seen in Fig. 3. Table II provides the obtained results for $M=4$, $l=1 . . M$ and $c=0.0 . .1 .0$ and Table III for $M=2 . .3$. Figs. 5,6,7 show them as function of $c$. For $c=1.0$ this basically means there is effectively only one usable stream. The mean is easily obtained as in Eq. 8 with Euler constant $\gamma=0.5772156649015328606065$.

$$
E\{\Delta S I N R(l)\}=\mu(l)+\sigma(l) * \gamma
$$

\section{NumericAl REsults}

In this example we assume single-user MIMO with $M=$ $N=4 \mathrm{Tx}$ and $\mathrm{Rx}$ antennas and also provide SISO results for comparison. See [11] for more details on this model.

TABLE IV

SECTORS AND ANTENNA DIRECTIVITY

\begin{tabular}{|c|c|c|}
\hline Sectors & 3 & 6 \\
\hline Antenna aperture horizontal $\theta_{3 d B}$ & $70^{\circ}$ & $35^{\circ}$ \\
Antenna aperture vertical $\phi_{3 d B}$ & $15^{\circ}$ & $15^{\circ}$ \\
\hline
\end{tabular}

Figure 9 shows results over the cell area for the IMTAdvanced scenarios defined in table $\mathrm{V}$ and [8]. The results use the analytic model of section II and III and did not use any simulation. The interference of neighbor cells and 


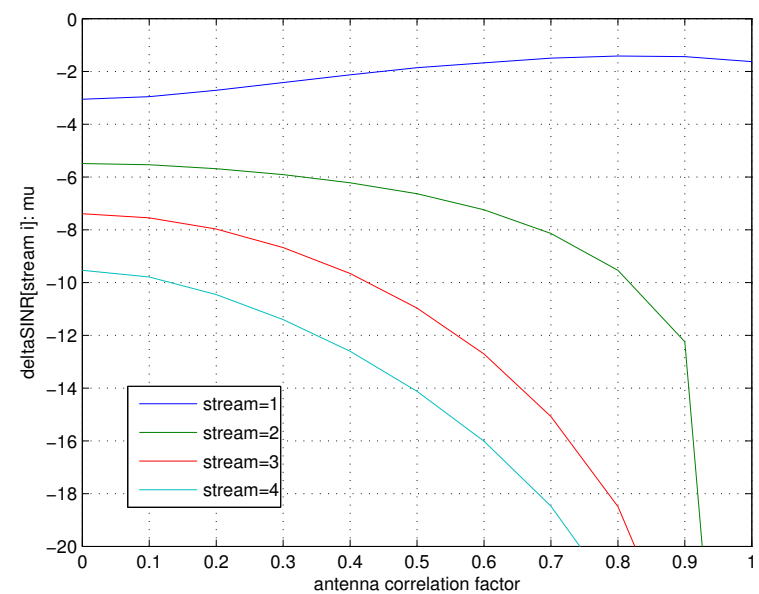

Fig. 5. Extreme value distribution parameter $\mu_{\Delta S I N R, l}=f(c, l)$

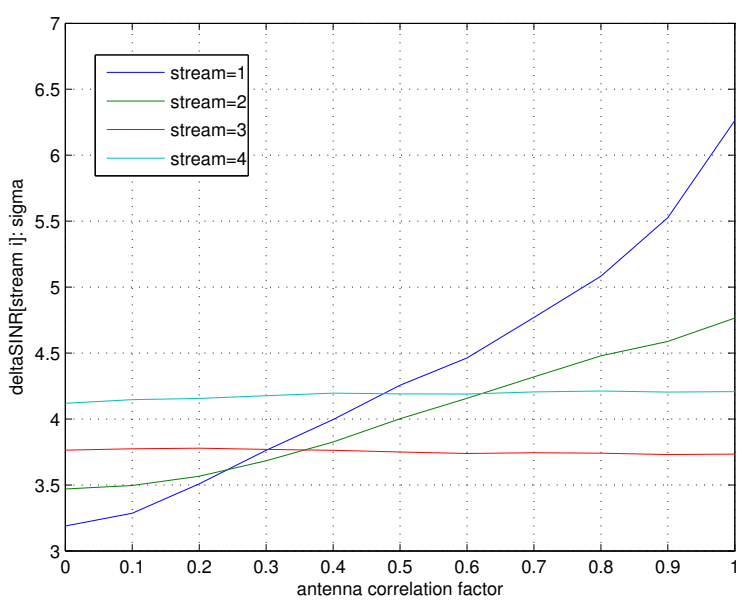

Fig. 6. Extreme value distribution parameter $\sigma_{\Delta S I N R, l}=f(c, l)$

sectors with the same layout is properly taken into account with a frequency reuse of one (most dense package). The DL SINR results plotted over the cell area show the $S I N R$ of the best station (BS,RN), not the maximum $S I N R$. Relays are assumed on half the distance to the cell border. The rate/throughput results contain the maximum achievable rate at a certain position within the cell, taking also the required first hop resources into account.

In Table VII the spectral efficiency results are given. $3 S, 6 S$ means 3 or 6 sectors, respectively. MIMO $-c$ means MIMO with cochannel correlation $c$. The number of relays is indicated by $0 R N, 3 R N, 6 R N$.

From Table VII can be seen that Relays and MIMO both give a performance benefit. Even if the cochannel correlation is $c=80 \%$. From Table II we can conclude that MIMO operates only well in areas of high $S I N R$. For relays we know that they are best located in areas of otherwise low $S I N R$. So we suggest a combination of both as they are not mutually exclusive. In a real system there must be a scheduler which selects MIMO where the $S I N R$ and channel correlation are suitable and otherwise switches back to SISO with receive diversity combining. Table II can also be used to determine these switching points.

For a system simulator [17] the actual channel condition

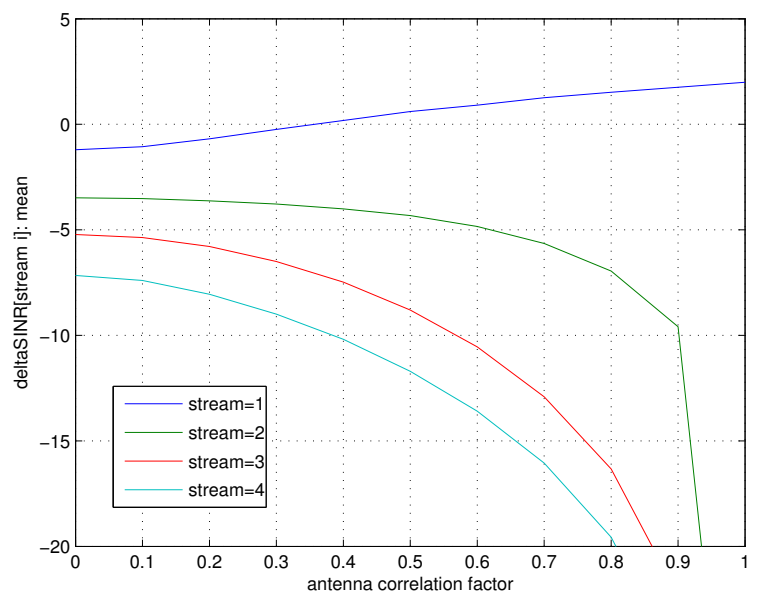

Fig. 7. Extreme value distribution mean: $E\{\Delta S I N R(l)\}=f(c, l)$

TABLE V

IMT-ADVANCED SCENARIO SPECIFICATIONS

\begin{tabular}{|c|c|c|c|c|}
\hline Scenario & $\begin{array}{c}\text { Urban } \\
\text { micro } \\
\text { UMi }\end{array}$ & $\begin{array}{c}\text { Urban } \\
\text { macro } \\
\text { UMa }\end{array}$ & $\begin{array}{c}\text { Suburban } \\
\text { macro } \\
\text { SMa }\end{array}$ & $\begin{array}{c}\text { Rural } \\
\text { macro } \\
\text { RMa }\end{array}$ \\
\hline$d_{B S-B S}$ & $200 \mathrm{~m}$ & $500 \mathrm{~m}$ & $1299 \mathrm{~m}$ & $1732 \mathrm{~m}$ \\
\hline$h_{B S}$ & $10 \mathrm{~m}$ & $25 \mathrm{~m}$ & $35 \mathrm{~m}$ & $35 \mathrm{~m}$ \\
\hline$r_{\min }$ & $10 \mathrm{~m}$ & $25 \mathrm{~m}$ & $35 \mathrm{~m}$ & $35 \mathrm{~m}$ \\
\hline Ant. tilt $\phi_{t}$ & $-12^{\circ}$ & $-12^{\circ}$ & $-6^{\circ}$ & $-6^{\circ}$ \\
\hline$f_{C}[G H z]$ & 2.5 & 2.0 & 2.0 & 0.8 \\
\hline$P_{T x}$ & $44 \mathrm{dBm}$ & $49 \mathrm{dBm}$ & $49 \mathrm{dBm}$ & $49 \mathrm{dBm}$ \\
\hline
\end{tabular}

must be generated frame-by-frame, signaled back from $U T$ to $B S$ and used as CSI for the scheduler. If the signaling round-trip-time is modeled precisely, then the channel must have a correlation in time, otherwise the scheduling decision is based on outdated information.

\section{CONCLUSION}

This paper presented a simple MIMO performance model suitable for system-level analysis or simulation tasks. These performance studies (e.g. for the IMT-Advanced systems) are very time-consuming and urgently need more simplified abstractions of the OSI layers below the area of interest. For the study of MAC layer algorithms (e.g. schedulers) there is no need for a sophisticated multi-dimensional channel model and this would only slow down the evaluation process. The method proposed here requires very little computation and is still open for extension to more elaborate statistics, e.g. correlation in frequency and/or time.

An application example featuring an IMT-Advanced scenario provides reasonable results and gives quantitative numbers for the spectral efficiency of relevant cases.

Future work will be the extension to asymmetric antenna configurations and a MIMO scenario model with distance dependent correlation properties $c(d)$.

\section{REFERENCES}

[1] J. Monserrat, P. Sroka, G. Auer, J. Cabrejas, D. Martin, A. Mihovska, R. Rossi, A. Saul, and R. Schoenen, "Advanced radio resource management for IMT-Advanced in WINNER+ (II)," in Proc. ICTMobileSummit 2010, Florence, Italy, Jun 2010.

[2] R. Pabst, B. Walke, D. C. Schultz, H. Yanikomeroglu et al., "Relaybased deployment concepts for wireless and mobile broadband radio," IEEE Communications Magazine, pp. 80-89, Sep 2004. 


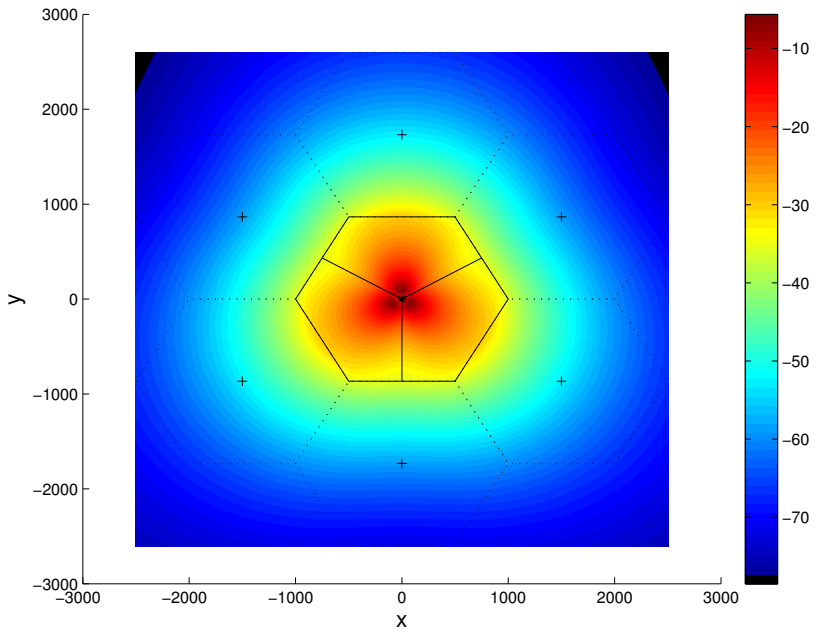

(a) RxPower/dBm with 3 sectors and directional antennas

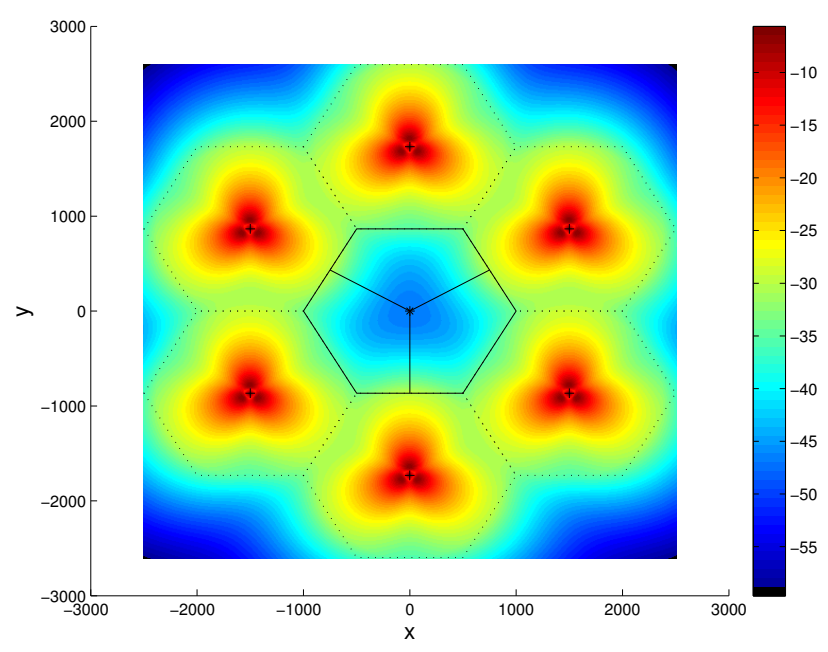

(b) Interference Power (dBm) of the scenario left

Fig. 8. Example Scenario (RMa) of the IMT-Advanced evaluation. Interference assumed worst case (all subchannels used)

TABLE VI

TECHNOLOGY PARAMETERS ACCORDING TO LTE-A

\begin{tabular}{|c|c|}
\hline Bandwidth [MHz] & FDD: 20DL,20UL \\
\hline Traffic & full load; best effort \\
\hline Antenna gain (boresight) & $17 d \mathrm{Bi}$ \\
\hline Thermal noise & $-174 d \mathrm{Bm} / \mathrm{Hz}$ \\
\hline UT noise figure & $5 d B$ \\
\hline
\end{tabular}

TABLE VII

SPECTRAL EFFICIENCY RESULTS FOR THE SCENARIO EVALUATION $[\mathrm{bit} / \mathrm{s} / \mathrm{Hz} /$ Sector $]$, GROSS WITHOUT MAC FRAME OVERHEAD

\begin{tabular}{|c|c|c|c|c|}
\hline Scenario & UMi & UMa & SMa & RMa \\
\hline 3S,SISO,0RN & 1.567 & 1.254 & 1.234 & 1.974 \\
\hline 3S,SISO,3RN & 1.945 & 1.804 & 1.825 & 2.310 \\
\hline 3S,MIMO-0.0,0RN & 3.971 & 2.871 & 2.853 & 5.740 \\
\hline 3S,MIMO-0.8,0RN & 3.525 & 2.875 & 2.906 & 5.009 \\
\hline 6S,SISO,0RN & 1.336 & 1.184 & 1.160 & 1.626 \\
\hline 6S,SISO,6RN & 1.581 & 1.927 & 1.996 & 1.781 \\
\hline 6S,MIMO-0.0,0RN & 2.780 & 2.453 & 2.564 & 3.762 \\
\hline
\end{tabular}

[3] M. Salem, A. Adinoyi, M. Rahman, H. Yanikomeroglu, D. Falconer, Y.-D. Kim, E. Kim, and Y.-C. Cheong, "An overview of radio resource management in relay-enhanced OFDMA-based networks," IEEE Communications Surveys and Tutorials, vol. 12, no. 3, Q3 2010.

[4] H. Yanikomeroglu, "Fixed and mobile relaying technologies for cellular networks," in Proc. of ASWN'02, Jul 2002, pp. 75-81.

[5] Z. Xie and B. Walke, "Resource allocation and reuse for inter-cell interference mitigation in ofdma based communication networks," in The 5th Annual International Wireless Internet Conference (WICON 2010), Singapore, Mar 2010, p. 6. [Online]. Available: http://www.comnets.rwth-aachen.de

[6] V. Jungnickel, L. Thiele, M. Schellmann, T. Wirth, W. Zirwas, T. Haustein, and E. Schulz, "Implementation Concepts for Distributed Cooperative Transmission," in 42nd Asilomar Conference on Signals, Systems and Computers, Monterey, USA, Oct. 2008.

[7] M. Kiiski, "LTE-Advanced: The Mainstream in Mobile Broadband Evolution," in Proceedings of the EW'2010, Lucca, It, Apr 2010, p. 6.

[8] ITU, "Report ITU-R M2135; Guidelines for evaluation of radio interference technologies for IMT-Adcanced", 2008.

[9] P. Smith and M. e. a. McKay, "Random Matrix processes: Quantifying Rates of Change in MIMO Systems," in Proceedings of the EW'2010, Lucca, It, Apr 2010, p. 6.

[10] R. Schoenen, W. Zirwas, and B. Walke, "Capacity and Coverage Analysis of a 3GPP-LTE Deployment Scenario," in Proceedings of the BWAW'08 colocated with ICC'08, Beijing, China, May 2008.

[11] R. Schoenen and C. Teijeiro, "System Level Performance Evaluation of LTE with MIMO and Relays in Reuse-1 IMT-Advanced Scenarios," in Proceedings of the IEEE WiCom, Chengdu, China, Sep 2010.

[12] R. Schoenen and F. Qin, "A Closed Loop Control Model separating Resource and Packet Scheduling in Multihop Cellular Networks," in Proc. of the European Wireless, Lucca, Italy, Apr 2010.

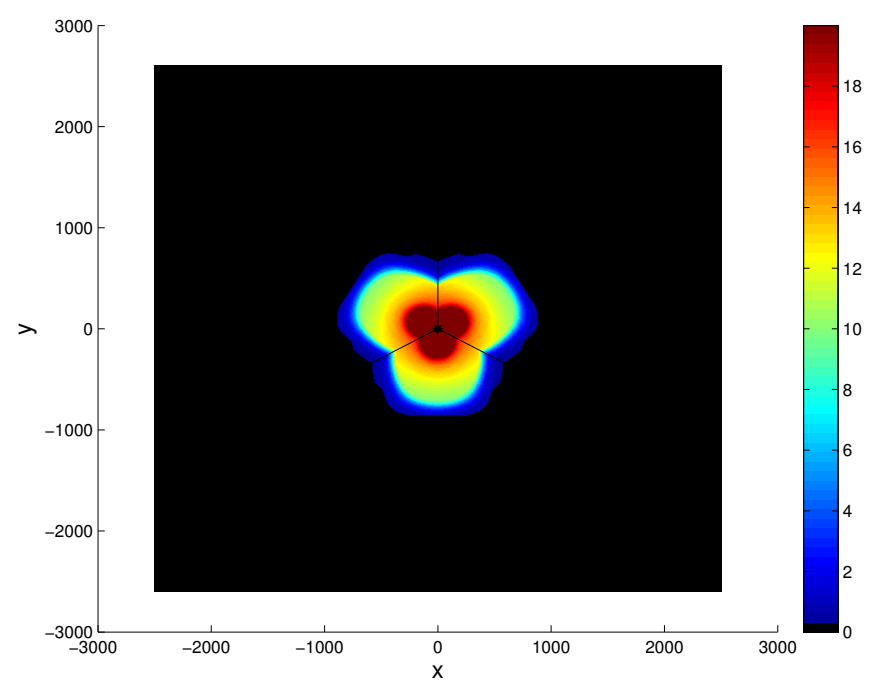

Fig. 9. Area results $M I(x, y)$ for the IMT-Advanced scenario RMa

[13] K. Brueninghaus and D. e. a. Astely, "Link performance models for system level simulations of broadband radio access systems," in Proceedings of the 17th PIMRC'2005, Berlin, Sep 2005, pp. 23062311.

[14] R. Schoenen and B. Walke, "On PHY and MAC performance of 3GLTE in a multi-hop cellular environment," in Proc. IEEE WiCom 2007, Shanghai, China, Sep 2007.

[15] A. Paulraj, R. Nabar, and D. Gore, Introduction to space-time wireless communications. Cambridge: Cambridge University Press, 2003.

[16] J. Mirkovic and B. Walke, "On performance of MIMO link adaptation in the presence of channel uncertainty," in Proceedings of 4th IEEE International Conference on Wireless and Mobile Computing, Networking and Communications 2008, Avignon, France, Oct 2008, p. 6. [Online]. Available: http://www.comnets.rwth-aachen.de

[17] S. Max, D. Bültmann, R. Jennen, and M. Schinnenburg, "Evaluation of imt-advanced scenarios using the open wireless network simulator," in Proceedings of the 3rd International ICST Conference on Simulation Tools and Techniques (SIMUTools 2010). Torremolinos, Malaga, Spain: ICST, Mar 2010. 